\title{
Phonon-induced Floquet topological phases protected by space-time symmetries
}

\author{
Swati Chaudhary,,$^{1, *}$ Arbel Haim, ${ }^{1,2, \dagger}$ Yang Peng, ${ }^{3,1,2, \star}$ and Gil Refael ${ }^{1}$ \\ ${ }^{1}$ Institute of Quantum Information and Matter and Department of Physics, California Institute of Technology, \\ Pasadena, California 91125, USA \\ ${ }^{2}$ Walter Burke Institute for Theoretical Physics, California Institute of Technology, Pasadena, California 91125, USA \\ ${ }^{3}$ Department of Physics and Astronomy, California State University, Northridge, California 91330, USA
}

(Received 15 January 2020; revised 6 October 2020; accepted 11 December 2020; published 29 December 2020; corrected 28 January 2021)

\begin{abstract}
For systems with spatial and nonspatial symmetries, the topological classification depends not only on these symmetries but also on the commutation/anticommutation relations between spatial and nonspatial symmetries. The coexistence of spatial and nonspatial symmetries together with appropriate commutation/anticommutation relations between them can give rise to crystalline and higher-order topological phases, which host gapless boundary modes. Alternatively, space-time symmetries in a Floquet system can take the role of spatial symmetries in deciding the topological classification. Promoting a spatial symmetry to a space-time symmetry can alter the commutation relations, which in turn can modify the topological properties of the system. We show how a coherently excited phonon mode can be used to promote a spatial symmetry with which the static system is always trivial to a space-time symmetry which supports a nontrivial Floquet topological phase. We demonstrate this effect by considering two systems: The first is a second-order topological superconductor, and the second is a first-order crystalline topological insulator. In both these cases, a coherently excited phonon mode is responsible for promoting the reflection symmetry to a time-glide symmetry. This newly introduced symmetry allows the previously trivial system to host gapless modes. In the first case, these are protected corner modes, while in the second case, these are gapless edge modes.
\end{abstract}

DOI: 10.1103/PhysRevResearch.2.043431

\section{INTRODUCTION}

The topology of electronic band structures of crystals is largely restricted by the existing symmetries [1-8], and its nontriviality is reflected in the presence of gapless modes located at the crystal boundaries [9-13]. For example, in topological insulators [14-16], where the band topology respects only nonspatial symmetries, such as the time-reversal, particle-hole, and chiral symmetries, the boundary modes are of codimension one (the codimension is the difference between the bulk dimension and the dimension along which the gapless modes propagate).

The presence of spatial symmetries can enrich the existing topological classification further and can also modify the conventional bulk-boundary correspondence. For example, recently, systems [17-25] were theoretically proposed to support gapless modes of higher codimensions, because of the additional spatial symmetries coexisting with the nonspatial ones. The order of such higher-order topological insulators is given by the codimension of the boundary modes. On

\footnotetext{
*swatich@ caltech.edu

†arbelh@caltech.edu

†yang.peng@csun.edu
}

Published by the American Physical Society under the terms of the Creative Commons Attribution 4.0 International license. Further distribution of this work must maintain attribution to the author(s) and the published article's title, journal citation, and DOI. the experimental side, codimension-two boundary modes are observed mostly in metamaterials, such as electric circuits [26] and photonic [27] and phononic [28-30] systems. The electronic second-order topological insulator is only realized in bismuth [31].

If a spatial symmetry coexists with nonspatial symmetries, the symmetry operator of the former can either commute or anticommute with the ones of the latter $[7,8]$. Therefore the coexistence of a certain spatial symmetry alone is not enough to guarantee the possibility of having a nontrivial band topology; appropriate commutation or anticommutation relations between the spatial and nonspatial symmetry operators are also needed.

Very recently, it was demonstrated that in a periodically driven system, a new space-time symmetry, such as time-glide or time-screw, can emerge, if the system is invariant under reflection or twofold rotation, together with a half-period time translation [32]. As far as topological classification is concerned, such a space-time symmetry can lead to a nontrivial Floquet band topology, in the same way as its spatial counterpart does in a static system, except for a possible alternation of the commutation/anticommutation relations with respect to the nonspatial symmetries [33,34].

This result leads to the following interesting question. When the commutation/anticommutation relation alternation does occur, is it able to periodically drive an initially topological trivial system, whose spatial symmetry does not have appropriate relations with respect to the nonspatial symmetries, into a nontrivial Floquet topological phase? 
In this paper, we answer this question by considering phonon-assisted space-time engineering, which promotes the spatial symmetry (such as reflection) of a static system into a space-time symmetry (such as time-glide), without changing the symmetry operator. In this way, the relations with respect to the nonspatial symmetries that are inappropriate for the spatial symmetry would become otherwise appropriate for the space-time symmetry.

\section{PHONON-ASSISTED SPACE-TIME ENGINEERING}

One assumption in the electronic band structure of a crystal is that the lattice is rigid, with ions fixed to their equilibrium positions. The success of this assumption in characterizing lots of properties of materials is that the energy due to lattice vibrations or phonons is much smaller compared with the electronic energy at the equilibrium lattice configuration.

However, it is known that a coherently excited and macroscopically occupied phonon mode can result in ions moving collectively [35]. When the material is in such a state, the electrons will experience a periodically oscillating ionic potential which can no longer be neglected. Indeed, it has been proposed in some recent works [36,37] that a phonon drive can be used to tune the topological features of graphene.

It is known that the symmetries of a crystal in equilibrium with a rigid lattice configuration are described by the space group of the lattice. The normal modes of the lattice vibrations, namely, the phonons, form the irreducible representations of this group. To be more specific, consider an order-two point-group operation $\hat{g}$ which squares to identity; the phonon modes must have a definite parity under this operation. Whereas the oscillating potential generated by the evenparity phonon is invariant under such a point-group operation at arbitrary times, the one generated by an odd-parity phonon breaks this point-group symmetry. Nevertheless, the timedependent potential $V(\boldsymbol{r}, t)$ generated by the latter acquires the space-time symmetry, given by $V(\hat{g} \boldsymbol{r}, t)=V(\boldsymbol{r}, t+T / 2)$, where $\boldsymbol{r}$ and $t$ are the spatial and temporal coordinates and $T$ is the oscillation period for the phonon. Hence we have managed to promote a spatial order-two symmetry described by $\hat{g}$, to a space-time symmetry described by the same operator, by coherently exciting a phonon mode that is odd under $\hat{g}$. This is an example of phonon-assisted space-time engineering.

In this paper, we provide two examples in which by promoting a spatial symmetry with operator $\hat{g}$ to a space-time symmetry, the commutation relations between $\hat{g}$ and nonspatial symmetries become appropriate for supporting a nontrivial (Floquet) topological phase, whereas only a trivial phase exists in a $\hat{g}$-symmetric static system. In the first case, adding a phonon drive gives rise to corner Majorana modes, and in the second case, it results in a quantized conductance as shown in Fig. 1. In Appendix A, we list all possibilities of realizing a topological nontrivial Floquet phase from a static trivial system by such phonon-assisted space-time engineering.

\section{TWO-DIMENSIONAL SYSTEM IN CLASS D OR BDI}

It is known that in the presence of reflection symmetry, class $\mathrm{D}$ or BDI exhibits topological behavior characterized by a mirror topological invariant whenever the reflection operator (a)

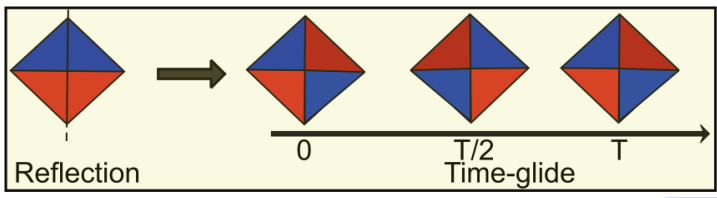

(b)

(c)

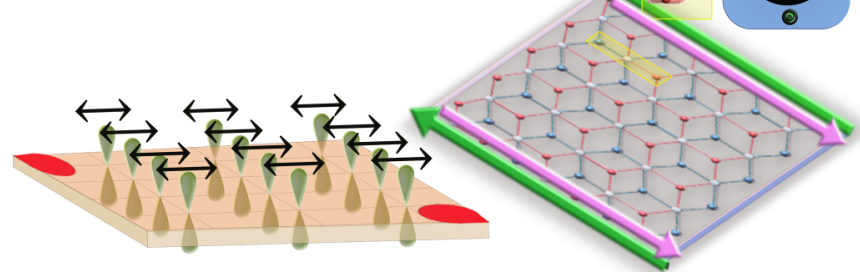

FIG. 1. Phonon-assisted space-time engineering. (a) Promotion of mirror symmetry to a time-glide symmetry. (b) A schematic of our proposal for class $\mathrm{D}$, where a phonon mode, shown by black arrows, can give rise to Floquet corner modes, shown by red dots. (c) Similarly, in class AIII, a phonon mode, shown in the yellow inset, can give rise to Floquet edge modes which result in a quantized conductance in a sample of bilayer graphene.

(described by $M$ ) commutes with the particle-hole operator $C$ (Table II in Appendix A). We demonstrate how a phonon drive can turn a trivial static system with $\{C, M\}=0$ into a nontrivial Floquet topological phase.

Consider a tight-binding model with nearest-neighbor hopping, $t_{0}$, on a two-dimensional square lattice placed in proximity to an $s$-wave superconductor described by the Bloch Hamiltonian

$$
H_{0}(\mathbf{k})=\left(m_{0}-2 t_{0} \cos k_{x}-2 t_{0} \cos k_{y}\right) \tau_{z}+\Delta \tau_{x}+b \sigma_{x},
$$

where $\sigma$ and $\tau$ are Pauli matrices acting on spin and particle-hole degrees of freedom, respectively. Here, we have expressed a Bogoliubov-de Gennes (BdG) Hamiltonian using a Nambu spinor basis $\left(c_{\uparrow}^{\dagger}, c_{\downarrow}^{\dagger}, c_{\downarrow},-c_{\uparrow}\right)$. In this basis, the last term, $b \sigma_{x}$, arises from a magnetic field in the $x$ direction, and particle-hole symmetry is given by $C=\tau_{y} \sigma_{y}$. This Hamiltonian has a reflection given by $M=\sigma_{x}$ flipping the coordinates in the $y$ direction. This also has a time-reversal symmetry given by $T=\mathbb{I}$, but it is not relevant for our purpose as the commutation relation with the time-reversal operator cannot be altered. Let us consider the effect of a reflection-symmetrybreaking phonon which produces a time-dependent Rashba spin-orbit coupling (SOC) given by

$$
H(\mathbf{k}, t)=2 \alpha_{0} \cos \omega t\left(\sin k_{x} \sigma_{y}-\sin k_{y} \sigma_{x}\right) \tau_{z} .
$$

An example of a phonon mode generating such a term is described in the next section.

The full Hamiltonian $H_{0}(\mathbf{k})+H(\mathbf{k}, t)$ has a time-glide symmetry with reflection $M=\sigma_{x}$, promoted from the static reflection symmetry. The role of this periodic drive in topological behavior can be understood better by considering the frequency-domain formulation of the Floquet problem restricted to two Floquet zones [33,34]. This $2 \times 2$ enlarged Hamiltonian reads

$$
\mathscr{H}(\mathbf{k})=\left(\begin{array}{cc}
H_{0}(\mathbf{k})+\frac{\omega}{2} & H_{1} \\
H_{\overline{1}} & H_{0}(\mathbf{k})-\frac{\omega}{2}
\end{array}\right),
$$

where $H_{n}=\frac{1}{T} \int_{0}^{T} H(t) e^{-i n \omega t} d t$ and $H_{0}$ is described in Eq. (1). 

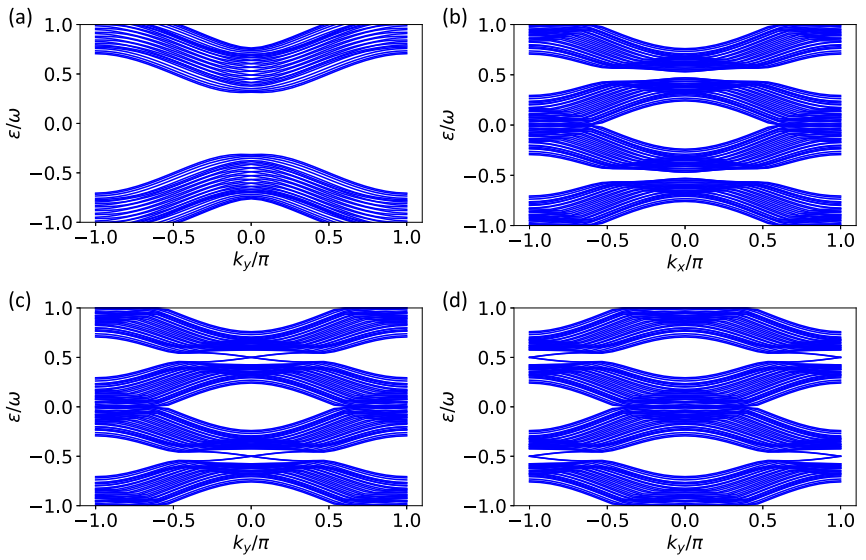

FIG. 2. Band structure for $m_{0}=\omega / 2+1, \Delta=0.9, \omega=4.8$, $L=15, b=0.15$, and $\alpha_{0}=0.5$, with periodic boundary conditions (a) in the $y$ direction for a static Hamiltonian, (b) in the $x$ direction for a Floquet Hamiltonian, and (c) and (d) in the $y$ direction for a Floquet Hamiltonian. In (d), $m_{0}=-\omega / 2-1$, and this change of sign results in a shift in the position of the gapless mode from $k_{y}=0$ to $k_{y}=\pi$ $\left(\Delta=0.5, \alpha_{0}=1.0\right.$ is shown in Appendix B).

This Hamiltonian has particle-hole symmetry given by $\mathscr{C}=\sigma_{y} \tau_{y} \rho_{x}$ and reflection $\mathscr{R}=\sigma_{x} \rho_{z}$, where we have introduced a set of Pauli matrices $\rho_{x, y, z}$ for the new spinor degree of freedom in the extended Floquet basis. It belongs to class $\mathrm{D}$ and has appropriate commutation relations. Its topological behavior can thus be understood in terms of mirror topological invariant $\mathbb{Z}_{2}$ (see Appendix B). The resulting band structure is shown in Fig. 2 with periodic boundary conditions (BCs) in one direction. It features gapless modes around quasienergy $\omega / 2$ for periodic BCs in the $y$ direction whenever $\mathbb{Z}_{2}$ is nontrivial. Now, if we modify the boundary such that the reflection symmetry is broken everywhere except at corners, then the edge modes are gapped out with opposite mass terms on two sides of the corner. However, at reflection-symmetric corners, this mass term is zero, and hence the edge modes are replaced by the hinge modes as shown in Fig. 3. We would like to emphasize that in our scheme, gapless modes appear only around quasienergy $\omega / 2$, while in certain Floquet schemes $[38,39]$ this kind of corner mode can appear at both quasienergy zero and $\omega / 2$.

These corner modes are very robust to disorder and other perturbations. We study the effect of disorder by modifying
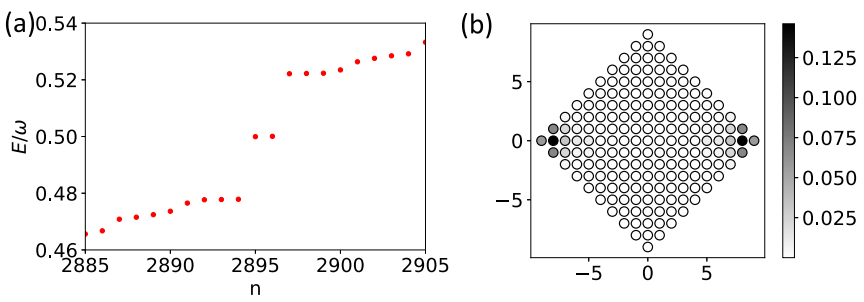

FIG. 3. (a) Energy spectrum of the Floquet Hamiltonian with $H_{1}$ of Eq. (2) around quasienergy $\omega / 2$ for open boundary conditions in both directions with reflection-symmetry-broken edges. (b) Support of the hinge mode for these boundary conditions corresponding to quasienergy $\omega / 2$.
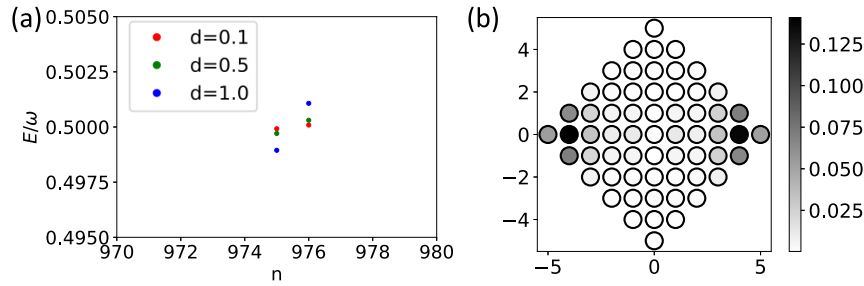

(c)

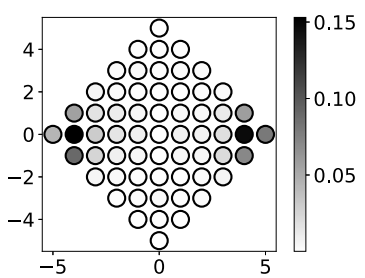

(d)

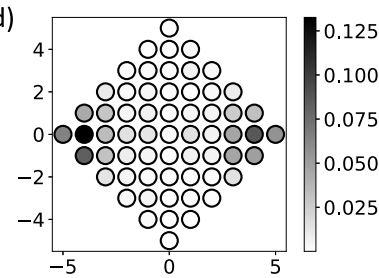

FIG. 4. (a) Spectra around $\omega / 2$ for three different values of disorder strength $d$. (b)-(d) Corner modes associated with these states for system size $L=6$. As $d$ increases, the corner start to smear out into the bulk.

each nearest-neighbor interaction $t \rightarrow t(1+h)$, where $h$ is a random number in the range of $\left[-\frac{d}{2}, \frac{d}{2}\right]$. When $d \ll 1$, we observe that the corner modes persist although there degeneracy is slightly lifted as shown in Fig. 4. These corner modes start to mix with bulk states only when the disorder becomes comparable to hopping amplitudes. However, this splitting becomes exponentially small for large-enough system size as shown in Fig. 5. In addition to disorder, these corner modes are very robust to other perturbations in hopping and sample boundaries (see Appendix D).

\section{A toy model for phonon-induced Rashba SOC}

The main ingredients needed for a Rashba SOC are the on-site $\pi-\sigma$ spin-dependent interactions and the nearestneighbor $\pi-\sigma$ hopping between same-parity orbitals. The spin-dependent on-site $\pi-\sigma$ interaction occurs naturally because of an $\mathbf{L} \cdot \mathbf{S}$ term, and the nearest-neighbor hopping between $\pi-\sigma$ orbitals of the same parity can be facilitated

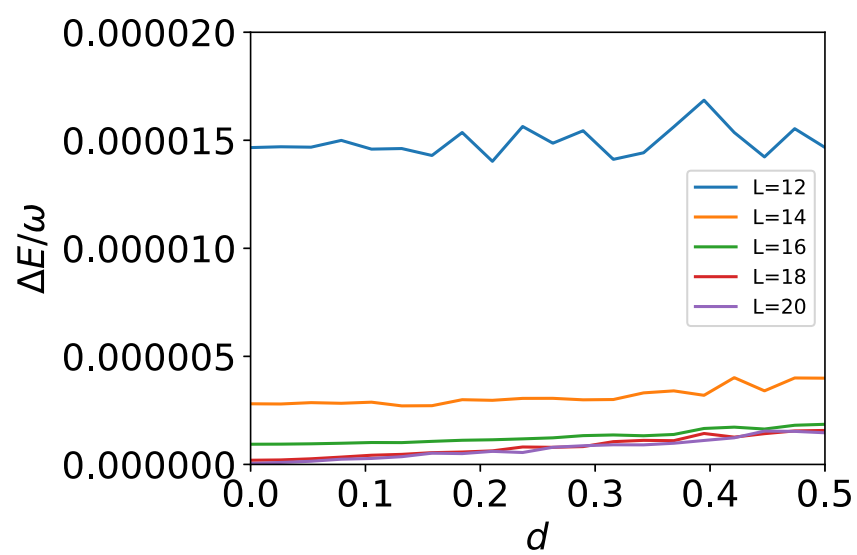

FIG. 5. Splitting of Majorana corner modes at quasienergy $\omega / 2$ as a function of disorder strength for different sample size obtained by averaging over 50 disorder realizations. These corner modes are obtained for open boundary conditions on a square-shaped sample of length $L$. 
TABLE I. Matrix element of $\mathbf{L} \cdot \mathbf{S}$ operator for $p$ orbitals.

\begin{tabular}{lccc}
\hline \hline Orbital & $p_{x}$ & $p_{y}$ & $p_{z}$ \\
\hline$p_{x}$ & 0 & $-i \zeta s_{z}$ & $i \zeta s_{y}$ \\
$p_{y}$ & $i \zeta s_{z}$ & 0 & $-i \zeta s_{x}$ \\
$p_{z}$ & $-i \zeta s_{y}$ & $i \zeta s_{x}$ & 0 \\
\hline \hline
\end{tabular}

by the opposite-parity orbitals or by ligands. For example, in graphene the intrinsic Rashba SOC occurs because of nearest-neighbor hopping between $p$ and $s / d$ orbitals [40,41]. Furthermore, the ligands can mediate a nearest-neighbor $\pi-\sigma$ hopping which depends on their position and thus can give rise to a time-dependent Rashba SOC when they oscillate. Consider that each site of our square-lattice model considered above has three nondegenerate $p$ orbitals where neighboring $p_{z}$ orbitals hybridize to form $\pi$ bands and the rest of the orbitals form $\sigma$ bands. These $\pi$ and $\sigma$ orbitals interact via $\mathbf{L} \cdot \mathbf{S}$ coupling (Table I). Furthermore, assume that an additional atom (which we call a ligand) with active $s$ orbitals is located between two lattice sites as shown in Fig. 6. This arrangement essentially forms a Lieb lattice. When the ligand is displaced in the $z$ direction, it induces a hopping between $\pi$ and $\sigma$ orbitals. When combined with on-site interaction $\mathbf{L} \cdot \mathbf{S}$ between $\pi$ and $\sigma$ orbitals, it produces an effective spindependent hopping between $\pi$ orbitals given by

$$
\begin{aligned}
\left\langle p_{z, i, j}\left|H_{\mathrm{eff}}\right| p_{z, i+1, j}\right\rangle & =t_{z z}+\frac{1}{\epsilon_{z x}} t_{i, i+1}^{z, x}\left\langle p_{x, i+1}|\mathbf{L} \cdot \mathbf{S}| p_{z, i+1}\right\rangle \\
& =t_{z z}+i \frac{t_{u} \zeta}{\epsilon_{z x}} \sigma_{y}
\end{aligned}
$$

where $t_{z z}=t_{0}$ is the direct hopping between two $p_{z}$ orbitals, $\epsilon_{z x}$ is the energy gap between $p_{z}$ and $p_{x}$ orbitals, $\zeta$ is SOC strength, and $t_{u}$ is ligand-induced hopping between $p_{z, i}$ and $p_{x, i+1}$.

Similarly, we can get a $\sigma_{x}$-dependent hopping in the $y$ direction. For a small lattice displacement, this ligand-induced hopping $t_{u} \approx\left(\frac{u(t)}{L} t_{s p \sigma}\right) t_{s p \sigma} / \epsilon_{s p}$, where $t_{s p \sigma}$ is the hopping between the $p_{x / y}$ orbital and the $s$ orbital of the ligand, $\epsilon_{s p}$ is the energy separation between the $s$ and $p$ orbitals, $L$ is the distance of the ligand from the neighboring lattice site, and $t_{s p \sigma} u(t) / L$ is the hopping between the $\pi$ orbital and $s$ orbital of the ligand, which comes into the picture only when the lattice displacement $u(t)$ is nonzero [42]. It gives rise to a time-dependent Rashba SOC which can be controlled by the lattice vibrations associated with the ligand motion. For a coherent phonon, $u(t) \approx u_{0} \cos \omega t$, where the ratio $u_{0} / L$ can be as large as 0.1 in certain cases $[43,44]$. The ligand-induced hopping $t_{u}$ depends on a lot of factors such as the phonon am-

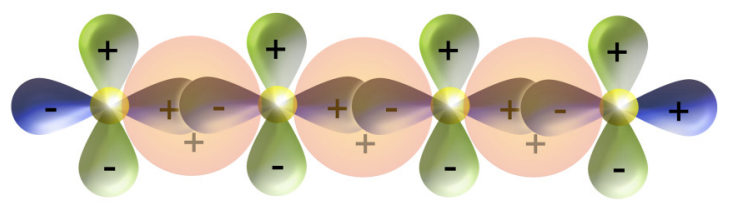

FIG. 6. $p_{z}$ (green) and $p_{x}$ (blue) orbitals of the two-dimensional square lattice along the $x$ direction with $s$ orbitals of the ligands shown in orange color. When the ligand ion is displaced in the $z$ direction, it induces a hopping between $p_{x}$ and $p_{z}$ orbitals.

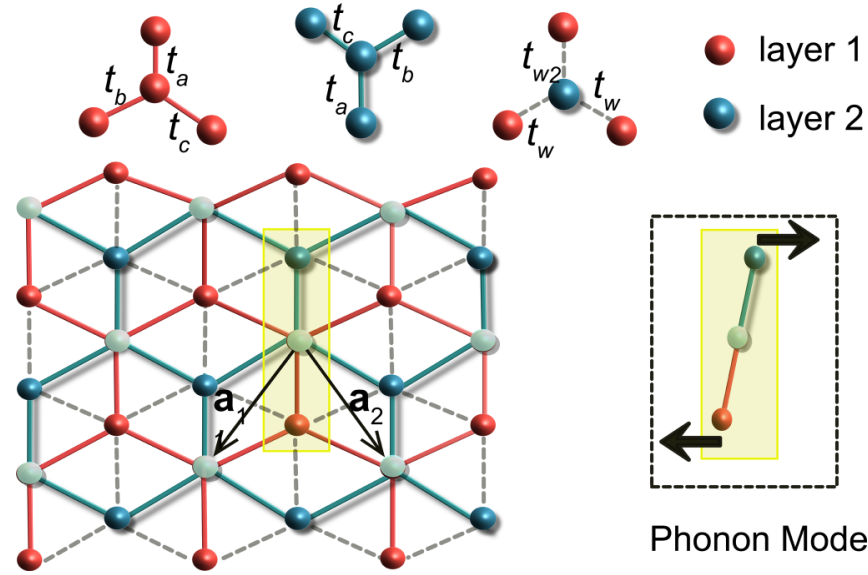

FIG. 7. A schematic for $A-B$ stacked bilayer honeycomb lattice with nearest-neighbor intralayer (solid lines) and interlayer (dashed lines) hopping. The unit cell of the triangular lattice is shown in a yellow box. The dimer sites (shown in light blue color) do not participate in any nearest-neighbor interlayer hopping. The right inset shows the phonon mode which affects the hopping parameters $t_{b}, t_{c}$, and $t_{w}$.

plitude, the energy separation $\epsilon_{s p}$, and the hopping $t_{s p \sigma}$, which can be much larger than the $\pi$ hopping $t_{z z}$. Depending on the ligand species, $\epsilon_{s p} \approx 1-10 \mathrm{eV}$, and thus the effective hopping $t_{s p \sigma}^{2} / \epsilon_{s p}$ can be anywhere between $0.1 t_{z z}$ and $5 t_{z z}$ since the ratio $t_{s p \sigma} / t_{z z} \approx 1-10$ usually. This rough estimate indicates that the ligand-induced hopping $t_{u}$ can be anywhere between $0.1 t_{z z}$ and $t_{z z}$, and thus the drive strength $\alpha_{0} \approx(0.1-1) t_{z z} \zeta / \epsilon_{z x}$ can be of the same order as $t_{z z}$ if SOC $\zeta$ is comparable to the energy separation between the $\pi$ and $\sigma$ orbitals.

\section{TWO-DIMENSIONAL SYSTEM IN CLASS AIII}

It is known that insulators in class AIII respecting the chiral symmetry (described by $S$ ) alone have only the trivial band topology. When a unitary reflection symmetry (described by $M$, with $M^{2}=1$ ) exists, a $\mathbb{Z}$ topological classification is possible if $[S, M]=0[7,8]$.

Consider a tight-binding model for a Bernal-stacked bilayer graphenelike lattice with nearest-neighbor intralayer hopping for all sites and nearest-neighbor interlayer hopping between nondimer sites as shown in Fig. 7. For periodic boundary conditions, the corresponding Bloch Hamiltonian reads

$$
\begin{aligned}
& H_{0}(\mathbf{k}) \\
& =t_{a} \tau_{x}+t_{3}\left(\tau_{x} \sigma_{x}+\sigma_{y} \tau_{y}\right) \\
& \quad+2 t_{b}\left(\cos \frac{k_{x}}{2} \cos \frac{\sqrt{3} k_{y}}{2} \tau_{x}+\cos \frac{k_{x}}{2} \sin \frac{\sqrt{3} k_{y}}{2} \tau_{y}\right) \\
& \quad+\left(t_{w} \cos \frac{k_{x}}{2} \cos \frac{\sqrt{3} k_{y}}{2}+\frac{t_{w_{2}}}{2} \cos \sqrt{3} k_{y}\right)\left(\sigma_{x} \tau_{x}-\sigma_{y} \tau_{y}\right) \\
& +\left(t_{w} \cos \frac{k_{x}}{2} \sin \frac{\sqrt{3} k_{y}}{2}+\frac{t_{w_{2}}}{2} \sin \sqrt{3} k_{y}\right)\left(\tau_{x} \sigma_{y}+\tau_{y} \sigma_{x}\right)
\end{aligned}
$$



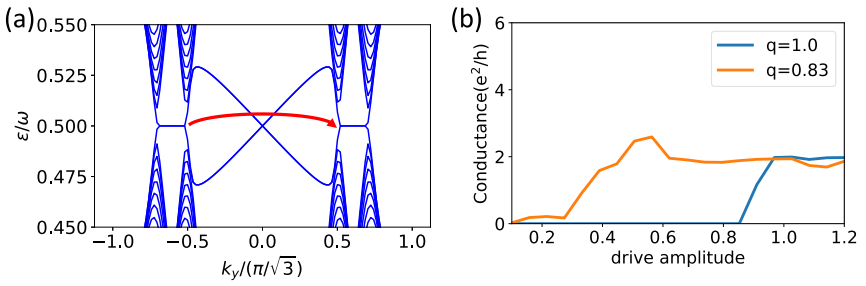

FIG. 8. (a) Band structure around $\omega / 2$ for the Floquet Hamiltonian (up to two Floquet sectors) of Eq. (C3), at $k_{y}=0$ for $t_{a}=2.4, t_{b}=1.2, t_{3}=0.5, \beta_{0}=0.1, t_{w}=0.5, \gamma_{0}=$ $0.5, \omega=4.4$, and $t_{w_{2}}=0.1$. Here, time-glide symmetry gives rise to two gapless edge modes which coexist with other gapless modes. (b) Conductance of a sample of width $W=51$ and length $L=61$ as a function of drive amplitude in the presence of a CDW perturbation; this perturbation suppresses the contribution of bulk modes, but the edge mode contribution survives. In this case, CDW $q \approx 0.8$ connects the two bulk gap closings as shown by the red arrow in (a).

where $\tau$ and $\sigma$ now operate on sublattice and layer degrees of freedom, respectively. It has chiral symmetry $S=\tau_{z}$ and mirror symmetry $M=\sigma_{x} \tau_{x}$ flipping the coordinates in the $y$ direction.

When a phonon mode is coherently excited such that the atoms $A_{1}$ and $B_{2}$ oscillate out of phase along the $x$ direction, the hopping for the nearest neighbors in the $\mathbf{a}_{1}$ and $\mathbf{a}_{2}$ directions as shown in Fig. 7 acquires an additional contribution

$$
\begin{aligned}
H(t)= & \beta(t) \sum_{\mathbf{r}_{i}, \alpha=1,2}\left(a_{\alpha, \mathbf{r}_{i}}^{\dagger} b_{\alpha, \mathbf{r}_{i}+\mathbf{a}_{1}}-a_{\alpha, \mathbf{r}_{i}}^{\dagger} b_{\alpha, \mathbf{r}_{i}+\mathbf{a}_{2}}+\text { H.c. }\right) \\
& +\gamma(t) \sum_{\mathbf{r}_{i}}\left(a_{1, \mathbf{r}_{i}}^{\dagger} b_{2, \mathbf{r}_{i}+\mathbf{a}_{1}}-a_{1, \mathbf{r}_{i}}^{\dagger} b_{2, \mathbf{r}_{i}+\mathbf{a}_{2}}+\text { H.c. }\right)
\end{aligned}
$$

and thus adds

$$
\begin{aligned}
H(\mathbf{k}, t)= & \beta(t)\left(-\sin \frac{k_{x}}{2} \cos \frac{\sqrt{3} k_{y}}{2} \tau_{y}+\sin \frac{k_{x}}{2} \sin \frac{\sqrt{3} k_{y}}{2} \tau_{x}\right) \\
& +\gamma(t) \sin \frac{k_{x}}{2} \cos \frac{\sqrt{3} k_{y}}{2}\left(\sigma_{x} \tau_{y}+\sigma_{y} \tau_{x}\right) \\
& +\gamma(t) \sin \frac{k_{x}}{2} \sin \frac{\sqrt{3} k_{y}}{2}\left(\sigma_{x} \tau_{x}-\sigma_{y} \tau_{y}\right)
\end{aligned}
$$

to $H_{0}(\mathbf{k})$, where $a$ and $b$ refer to the $A$ and $B$ sublattice sites, respectively, $\alpha$ indicates the layer index, and $\beta(t)$ and $\gamma(t)$ are proportional to the lattice displacement $u(t)$ for small phonon amplitudes. Their magnitude can be estimated as $\beta(t) \approx$ $\eta u(t) t_{0} / d_{0}$, where $\eta \approx 1-4, t_{0}$ is the static hopping between two sites, and $d_{0}$ is the equilibrium separation between two sites, as the hopping in a tight-binding model usually changes by a factor of $\left(\frac{d_{0}}{d_{0}+u}\right)^{\eta}$ [45]. For a coherent phonon, the lattice displacement $u(t) \approx u_{0} \cos \omega t$, which gives $\beta(t)=\beta_{0} \cos \omega t$, and thus $H(\mathbf{k}, t)=H_{1}(\mathbf{k}) \cos \omega t$. For a lattice displacement of $5-10 \%$, the drive strength $\beta_{0}$ and $\gamma_{0}$ can be anywhere between 5 and $40 \%$ of the static hopping amplitude $t_{0}$.

Now, the $2 \times 2$ enlarged Hamiltonian [Eq. (3)] has a chiral symmetry $\mathscr{S}=S \rho_{x}$ and a reflection symmetry realized by $\mathscr{R}=M \rho_{z}$. Since $[\mathscr{S}, \mathscr{R}]=0$, we can have a nontrivial classification with a mirror $\mathbb{Z}$ topological invariant. As shown in Fig. 8, in the nontrivial regime, it hosts gapless edge modes along $y$ boundaries for the driven system which are protected by reflection symmetry. These gapless edge modes at $k_{y}=0$ coexist with some gapless bulk modes at arbitrary $\pm k_{y}$ which are not protected by the reflection symmetry. These points arise due to bulk-band gap closings which can be gapped out by a charge density wave (CDW) of wavelength $q$. This perturbation is implemented by adding a term of the form of $\cos (q p)$ to all nearest-neighbor hoppings in the static and the drive parts for each site $\mathbf{R}=n \mathbf{a}_{1}+p \mathbf{a}_{2}$ on the underlying triangular lattice of bilayer graphene (see Appendix $\mathrm{C}$ for more details). This kind of perturbation results in a quantized conductance as shown in Fig. 8, where we have calculated the DC conductance numerically by using the Floquet scattering matrix approach [46]. We obtain this $S$ matrix using KWANT [47] for two leads by accounting for the photon degree of freedom using the Floquet representation of the Hamiltonian. In order to calculate the transmission coefficient, we sum up over incoming modes in the zeroth Floquet sector, which accounts for the absence of drive in leads. For the outgoing modes, we keep nonzero photon sectors to account for photon-assisted transport, which gives the transmission at energy $E$ :

$$
T(E)=\sum_{\alpha, \beta, n}\left|S_{\alpha, n ; \beta, 0}(E)\right|^{2},
$$

where $\alpha$ and $\beta$ represent the outgoing and incoming modes, respectively, and $n$ is the photon degree of freedom. Alternatively, the gapless bulk modes can also be removed by a drive-induced interlayer imaginary hopping between dimers, which makes it a Floquet higher-order topological phase (see Appendix C).

This establishes that the symmetry-promotion action of phonon drive can have much broader implications as it offers a possibility to produce a quantized conductance with a phonon drive in a previously trivial system.

\section{CONCLUSIONS}

We discussed how the symmetry-promotion action of a phonon drive can allow a previously trivial system to exhibit a topological classification. We presented two schemes where this can occur involving systems in class D and class AIII. These schemes require a phonon drive with a lattice displacement of the order of $0.1 \AA$. This can be achieved, for example, by exciting a particular phonon mode using ultrafast light pulses (see, for example, Ref. [48]). Usually, these phonons have a lifetime greater than $10 \mathrm{ps}$, which can allow a conductivity measurement with ultrafast devices based on terahertz operated photoconductive switches and scanning tunneling microscope (STM) tips [49,50], or even time-resolved angleresolved photoemission spectroscopy (ARPES) [51-55].

Both of these schemes need phonon frequencies to be of the same order as the hopping parameter. In most materials, electronic bandwidths are usually much greater than phonon frequencies. These frequencies are decided by the bond strength, while the hopping parameter in graphenelike lattices depends on overlap between neighboring $\pi$ orbitals. This kind of parameter regime can be realized by suppressing the hopping parameter without affecting the bond strengths between neighboring atoms, as the latter determine the phonon frequencies. Alternatively, one can consider placing the system on a substrate which binds to different sites such that 
the phonon frequencies are increased. These lattice models can be possibly realized using a covalent organic framework $[56,57]$ where molecular orbitals play the same role as atomic orbitals in our model and hopping parameters are of the order of $100 \mathrm{meV}$, and some of these materials can have phonon frequencies of the same order [58]. Similarly, twisted bilayer materials can provide another platform to realize the class AIII model where hopping parameters can be made comparable to certain phonon frequencies $[59,60]$.

\section{ACKNOWLEDGMENTS}

We acknowledge support from the Institute of Quantum Information and Matter, an NSF Physics Frontiers Center funded by the Gordon and Betty Moore Foundation, the Packard Foundation, and the Simons Foundation. A.H. and Y.P. are grateful for support from the Walter Burke Institute for Theoretical Physics at Caltech. Y.P. acknowledges sup- port from the startup fund from California State University, Northridge. G.R. is grateful for support from ARO MURI Award No. W911NF-16-1-0361, "Quantum Materials by Design with Electromagnetic Excitation," sponsored by the US Army.

\section{APPENDIX A: EFFECTIVE SYMMETRY OPERATORS AND MODIFIED COMMUTATION RELATIONS IN THE EXTENDED FLOQUET BASIS}

In this Appendix, we briefly review the effective symmetry operations for the frequency-domain representation of the time-periodic Hamiltonian when the system has a time-glide symmetry.

In this case, we show that the original time-glide symmetry of a time-periodic Hamiltonian is mapped to the reflection symmetry for the effective Hamiltonian [33]. The time-periodic Hamiltonian here follows:

$$
M H(\mathbf{k}, t) M^{-1}=H\left(-k_{x}, \mathbf{k}_{\|}, t+T / 2\right) .
$$

Here, we are interested in studying these systems in the Fourier basis and thus consider the above Hamiltonian in the extended Floquet basis given by

$$
H_{\text {full }}(\mathbf{k})=\left(\begin{array}{ccccccccc} 
& \multicolumn{1}{c}{H_{0}+2 \omega} & H_{1} & H_{2} & . & & & & \\
H_{\overline{1}} & H_{0}+\omega & H_{1} & H_{2} & & & \\
H_{\overline{2}} & H_{\overline{1}} & H_{0} & H_{1} & H_{2} & . & \\
\cdot & H_{\overline{2}} & H_{\overline{1}} & H_{0}-\omega & H_{1} & H_{2} & . & \\
& & H_{\overline{2}} & H_{\overline{1}} & H_{0}-2 \omega & H_{1} & H_{2} & . \\
& & & & & \cdot & &
\end{array}\right)
$$

and

$$
H_{n}(\mathbf{k})=\frac{1}{T} \int_{0}^{T} H(\mathbf{k}, t) e^{-i n \omega t} .
$$

In this basis, the time-glide symmetry is given by effective reflection

$$
\mathcal{R}_{\text {eff }}=\left(\begin{array}{cccccc}
\cdot & & & & & \\
& M & & & & \\
& -M & & & \\
& & M & & \\
& & & -M & \\
& & & & & \cdot
\end{array}\right),
$$

and other symmetry operators are also modified, for example, the new (effective) operators for time reversal, charge conjugation, and chiral symmetry are now given by

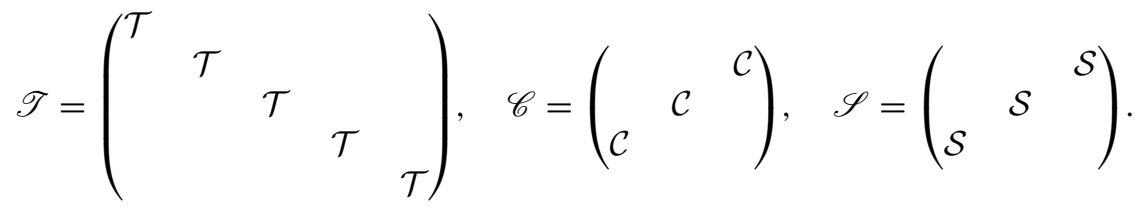

Now, the commutation relation between effective different symmetry operators is different from the commutation relation for the static case. This indicates that the effective Hamiltonian, although it belongs to the same Altland-
Zirnbauer (AZ) class as the static Hamiltonian, can allow the existence of a nontrivial topological phase (for, e.g., $\{M, S\}=$ $0 \Longrightarrow[\mathcal{R}, \mathscr{S}]=0$ ) in the presence of this time-glide symmetry. We are going to exploit this feature in realizing a 
TABLE II. Symmetry promotion from reflection $R$ and twofold rotation $C$.

\begin{tabular}{lcccc}
\hline \hline & & & & $3 \mathrm{D}$ \\
\cline { 2 - 4 } Class & \multicolumn{2}{c}{$2 \mathrm{D}$} & Symmetry promotion & $R_{+}$ \\
\hline AIII & Symmetry promotion & Classification & $R_{--}$ & $R_{-}$ \\
BDI & $R_{-}$ & $\mathbb{Z}$ & $R_{--}$ \\
D & $R_{+-}$ & $\mathbb{Z}$ & $R_{++}$ & $\mathbb{Z}$ \\
DIII & $R_{-}$ & $\mathbb{Z}_{2}$ & $R_{++}$ & $\mathbb{Z}$ \\
CII & $R_{+-}$ & $\mathbb{Z}_{2}$ & $C_{2-}$ \\
CI & $R_{+-}$ & $2 \mathbb{Z}$ & $C_{2+-}$ & $\mathbb{Z}_{2}^{2}$ \\
AIII & $R_{-+}$ & $2 \mathbb{Z}$ & $C_{2+-}$ \\
BDI & $C_{2+}$ & $\mathbb{Z}$ & $C_{2+-}$ \\
DIII & $C_{2++}$ & $\mathbb{Z}$ & $\mathbb{Z}^{2}$ \\
CII & $C_{2++}$ & $\mathbb{Z}$ & $C_{2+-}, C_{2-+}$ \\
CI & $C_{2++}$ & $2 \mathbb{Z}$ & $\mathbb{Z}$ \\
\hline \hline
\end{tabular}

nontopological phase by altering the modified commutation relations, which results in a change in topological classification as shown in Table II. Although in this paper we use a periodic drive to promote reflection symmetry to a time-glide symmetry in two dimensions (2D), the same ideas can be applied to twofold rotations and inversion symmetry in two and three dimensions, and the consequences of such promotions are discussed in Tables II and III.

When the drive is monochromatic, $H_{n}=0$ for $|n|>1$, and thus thewww above symmetries can be best described in terms of constraints on $H_{0}$ and $H_{1}$. In this case, the chiral-symmetric Hamiltonians satisfy

$$
\begin{aligned}
& S H_{0}(\mathbf{k}, \mathbf{r}) S^{-1}=-H_{0}(\mathbf{k}, \mathbf{r}), \\
& S H_{1}(\mathbf{k}, \mathbf{r}) S^{-1}=-H_{1}^{+}(\mathbf{k}, \mathbf{r}) .
\end{aligned}
$$

Similarly, particle-hole symmetry is given by

$$
\begin{aligned}
& C H_{0}^{*}(\mathbf{k}, \mathbf{r}) C^{-1}=-H_{0}(-\mathbf{k}, \mathbf{r}), \\
& C H_{1}^{*}(\mathbf{k}, \mathbf{r}) C^{-1}=-H_{1}^{+}(-\mathbf{k}, \mathbf{r}),
\end{aligned}
$$

and time-glide symmetry is given by

$$
\begin{aligned}
& M H_{0}\left(k_{x}, k_{y}\right) M^{-1}=H_{0}\left(-k_{x}, k_{y}\right), \\
& M H_{1}\left(k_{x}, k_{y}\right) M^{-1}=-H_{1}\left(-k_{x}, k_{y}\right) .
\end{aligned}
$$

\section{APPENDIX B: MIRROR TOPOLOGICAL INVARIANT $M \mathbb{Z}_{2}$ FOR CLASS D, $R_{+}$IN TWO DIMENSIONS}

For class D, when the particle-hole and reflection symmetry operator commute, the different topological phases can be

TABLE III. Symmetry promotion from inversion $I$.

\begin{tabular}{lcc}
\hline \hline & \multicolumn{2}{c}{$3 \mathrm{D}$} \\
\cline { 2 - 3 } Class & Symmetry promotion & Classification \\
\hline AIII & $I_{+}$ & $\mathbb{Z}^{2}$ \\
BDI & $I_{++}$ & $\mathbb{Z}$ \\
D & $I_{+}$ & $\mathbb{Z}$ \\
DIII & $I_{++}$ & $\mathbb{Z}^{2}$ \\
CII & $I_{--}$ & $\mathbb{Z}_{2}^{2}$ \\
CI & $I_{--}, I_{++}$ & $\mathbb{Z},(2 \mathbb{Z})^{2}$ \\
\hline \hline
\end{tabular}

distinguished on the basis of the mirror topological invariant $M \mathbb{Z}_{2}$. This invariant can be calculated at reflection-symmetric hyperplanes by first block-diagonalizing the Hamiltonian in the $\mathscr{R}$ basis and then calculating the $\mathbb{Z}_{2}$ invariant for one block. When the reflection operator commutes with the effective particle-hole operator $\mathscr{C}$, these two blocks do not mix, and hence a classification can be made on the basis of this topological invariant for one block. In the main text we considered the undriven model:

$$
H_{0}\left(k_{x}, k_{y}\right)=m_{1} \tau_{z}+\Delta \tau_{x}+b \sigma_{x},
$$

where $m_{1}=m-2 t_{0} \cos k_{x}-2 t_{0} \cos k_{y}$ with a drive of the form

$$
\begin{aligned}
H(t) & =2 \alpha_{0} \cos \omega t\left(\sin k_{x} \sigma_{y}-\sin k_{y} \sigma_{x}\right) \tau_{z} \\
& =H_{1} e^{i \omega t}+H_{1}^{\dagger} e^{-i \omega t} .
\end{aligned}
$$

This Hamiltonian has a particle-hole symmetry given by $C=$ $\tau_{y} \sigma_{y}$ and time-glide symmetry with reflection $M=\sigma_{x}$ about the $y$ axis $(y \rightarrow-y)$. We can cast it into a more familiar form if we use the eigenstate basis of $m(\mathbf{k}) \tau_{z}+\Delta \tau_{x}$, which corresponds to a transformation $\tau_{z} \rightarrow \cos \theta \tau_{z}-\sin \theta \tau_{x}$, where $\cos \theta=\frac{m(\mathbf{k})}{\sqrt{m(\mathbf{k})+\Delta^{2}}}$. In this basis,

$$
H_{0}=\sqrt{m(\mathbf{k})^{2}+\Delta^{2}} \tau_{z}+b \sigma_{x}
$$

and

$$
H_{1}=2 \alpha_{0}\left(\sin k_{x} \sigma_{y}-\sin k_{y} \sigma_{x}\right)\left(\frac{m(\mathbf{k})}{E_{k}} \tau_{z}-\frac{\Delta}{E_{k}} \tau_{x}\right),
$$

where $E_{k}=\sqrt{m(\mathbf{k})^{2}+\Delta^{2}}$. In this basis, the particle-hole operator $C=\tau_{y} \sigma_{y}$ and time-glide operator $M=\sigma_{x}$ remain the same. Now the Floquet Hamiltonian for photon sectors $n$ and 
$n+1$ can be written as

$$
H_{F}(\mathbf{k})=\left(\begin{array}{cc}
E_{k} \tau_{z}+\frac{\omega}{2} \mathbb{I}+b \sigma_{x} & \alpha_{0}\left(\sin k_{x} \sigma_{y}-\sin k_{y} \sigma_{x}\right)\left(\frac{m(\mathbf{k})}{E_{k}} \tau_{z}-\frac{\Delta}{E_{k}} \tau_{x}\right) \\
\alpha_{0}\left(\sin k_{x} \sigma_{y}-\sin k_{y} \sigma_{x}\right)\left(\frac{m(\mathbf{k})}{E_{k}} \tau_{z}-\frac{\Delta}{E_{k}} \tau_{x}\right) & E_{k} \tau_{z}-\frac{\omega}{2} \mathbb{I}+b \sigma_{x}
\end{array}\right) .
$$

The topological behavior of this Hamiltonian can also be understood by focusing on its inner $4 \times 4$ block given by

$$
\mathscr{H}(\mathbf{k})=\left(\begin{array}{cc}
\left(-E_{k}+\frac{\omega}{2}\right) \mathbb{I}+b \sigma_{x} & \alpha_{0}\left(\sin k_{x} \sigma_{y}-\sin k_{y} \sigma_{x}\right) \frac{\Delta}{E_{k}} \\
\alpha_{0}\left(\sin k_{x} \sigma_{y}-\sin k_{y} \sigma_{x}\right) \frac{\Delta}{E_{k}} & \left(E_{k}-\frac{\omega}{2}\right) \mathbb{I}+b \sigma_{x}
\end{array}\right)=\left(-E_{k}+\frac{\omega}{2}\right) \rho_{z}+b \sigma_{x}+\alpha_{0}\left(\sin k_{x} \sigma_{y}-\sin k_{y} \sigma_{x}\right) \frac{\Delta}{E_{k}} \rho_{x},
$$

where $\rho$ indicates the photon degree of freedom. In this case, the particle-hole symmetry $\mathscr{C}=\sigma_{y} p_{y}$, and it has a reflection symmetry $\mathscr{R}=\sigma_{x} \rho_{z}$ which commutes with $\mathscr{C}$; hence it can be characterized by a mirror $\mathbb{Z}_{2}$ invariant. In order to calculate this topological invariant, we go to reflection-symmetric hyperplanes $\left(k_{y}=0, \pi\right)$ and express this Hamiltonian in the basis of $M$ arranged such that the first block has eigenvalue +1 . The above Hamiltonian of Eq. (B6) takes the following form:

$$
\mathscr{H}\left(k_{x}\right)=\left(\begin{array}{cc}
H_{+} & \\
& H_{-}
\end{array}\right)=\left(\begin{array}{cccc}
-E_{k}+\frac{\omega}{2}+b & -i \alpha_{0} \sin k_{x} \frac{\Delta}{E_{k}} & 0 & 0 \\
i \alpha_{0} \sin k_{x} \frac{\Delta}{E_{k}} & E_{k}-\frac{\omega}{2}-b & 0 & 0 \\
0 & 0 & E_{k}-\frac{\omega}{2}+b & -i \alpha_{0} \sin k_{x} \frac{\Delta}{E_{k}} \\
0 & 0 & i \alpha_{0} \sin k_{x} \frac{\Delta}{E_{k}} & -E_{k}+\frac{\omega}{2}-b
\end{array}\right),
$$

with

$$
R=\left(\begin{array}{llll}
1 & & & \\
& 1 & & \\
& & -1 & \\
& & & -1
\end{array}\right) \text { and } P=\left(\begin{array}{llll}
-1 & & & \\
& & & 1
\end{array}\right)
$$

in the new basis. Now, each block belongs to class D, and in order to calculate the mirror $\mathbb{Z}_{2}$ invariant, we can pick any block. For example, if we pick $H_{+}$, then the invariant can be calculated from the Pfaffian of $H_{+}$at $k_{x}=0, \pi$. At $k_{y}=0$, the $\mathbb{Z}_{2}$ topological invariant is given by

$\eta_{k_{y}=0}=\operatorname{sgn}\left[\left(E_{k}-\frac{\omega}{2}+b\right)_{\left(k_{x}=0, k_{y}=0\right)}\left(E_{k}-\frac{\omega}{2}+b\right)_{\left(k_{x}=\pi, k_{y}=0\right)}\right]$.

Similarly, at $k_{y}=\pi$,

$\eta_{k_{y}=\pi}=\operatorname{sgn}\left[\left(E_{k}-\frac{\omega}{2}+b\right)_{\left(k_{x}=0, k_{y}=\pi\right)}\left(E_{k}-\frac{\omega}{2}+b\right)_{\left(k_{x}=\pi, k_{y}=\pi\right)}\right]$,

and thus the mirror $\mathbb{Z}_{2}$ invariant is now given by

$$
\eta_{M \mathbb{Z}_{2}}=1-\left|\eta_{k_{y}=0}-\eta_{k_{y}=\pi}\right|=\operatorname{sgn}\left[\eta_{k_{y}=0} \eta_{k_{y}=\pi}\right] \text {; }
$$

its dependence on $m_{0}$ and $\Delta$ is shown in Fig. 9. This indicates that if the system size is quite large, we can get a nontrivial phase for a very small value of superconducting gap $\Delta$, but for small system sizes we find the gapless edge modes only for $\Delta \approx 0.5$ as the bulk gap becomes very small for lower values of $\Delta$.

Although the topological invariant is nontrivial for a large range of $m$ and $\Delta$, the system does not seem to exhibit corner modes for small values of $\Delta$. In order to understand the regime for gapless boundary states, we study an eight-band model, which captures the essential features of the above model. This model is given by the Hamiltonian

$$
H_{\text {eff }}=\left(\begin{array}{cc}
H_{0}+\frac{\omega}{2} & H_{1} \\
H_{1}^{\dagger} & H_{0}-\frac{\omega}{2}
\end{array}\right) .
$$

The spectrum of this Hamiltonian is shown in Fig. 10 for open boundary conditions. In certain cases, the bulk gap becomes very small, and thus the gapless boundary modes or zeroenergy hinge modes cannot be observed for a small system size.

\section{APPENDIX C: EFFECT OF A CDW PERTURBATION AND A PHONON-INDUCED COMPLEX HOPPING TERM IN A CLASS AIII EXAMPLE}

\section{Effect of translation-symmetry-breaking perturbations on gapless bulk modes at Floquet zone boundaries}

We study the effect of a charge-density-wave-type perturbation in the bilayer graphene model considered above. For each site $\mathbf{R}=n \mathbf{a}_{1}+p \mathbf{a}_{2}$ on the underlying triangular lattice of bilayer graphene, we add a term of the form $A_{0} \cos (q p)$ to all the nearest-neighbor hopping in the static and the drive parts. We calculate the conductance in the $y$ direction for different amplitudes $A_{0}$ and the wave vector $q$ of this extra term (a)

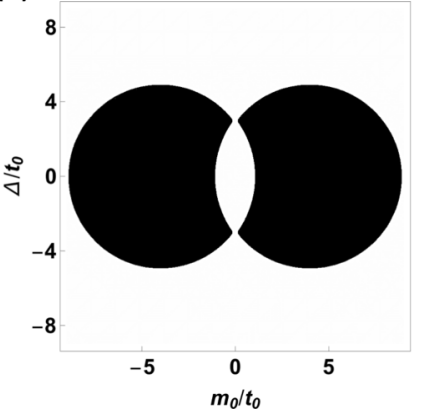

(b)

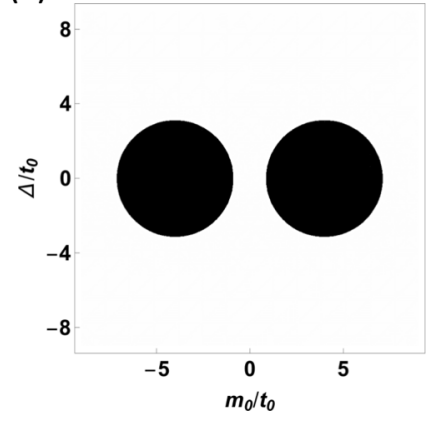

FIG. 9. Mirror topological invariant $\eta_{M \mathbb{Z}_{2}}$ as a function of parameters $m_{0}$ and $\Delta$ for $b=0.2 t_{0}$ and (a) $\omega=9.6 t_{0}$, (b) $\omega=3 t_{0}$. Black regions indicate the nontrivial region. 


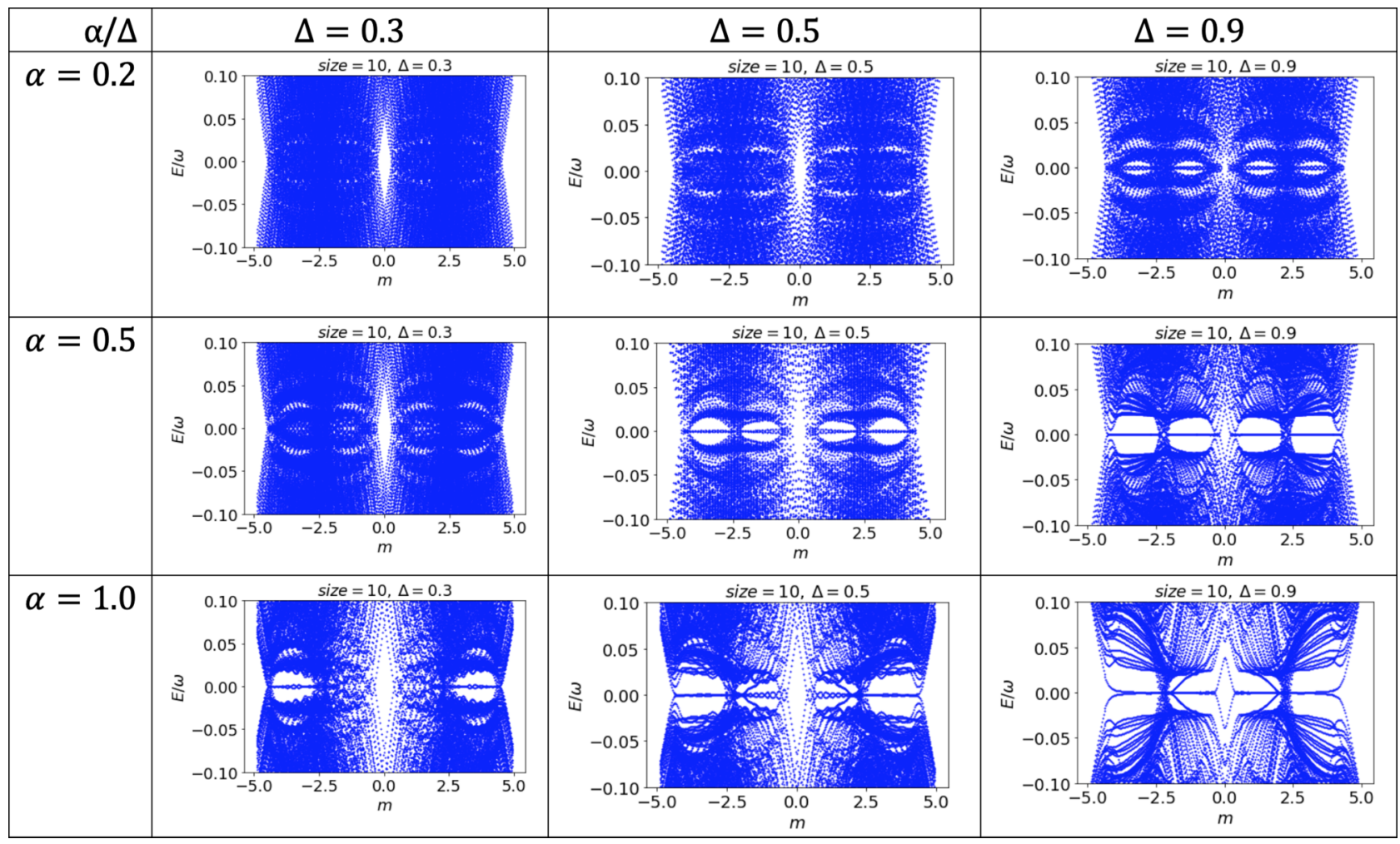

FIG. 10. Energy spectrum around zero energy as a function of $m$ for an eight-band model capturing the main features of the $\tau_{z}$ drive considered in Eq. (B12).

(a)

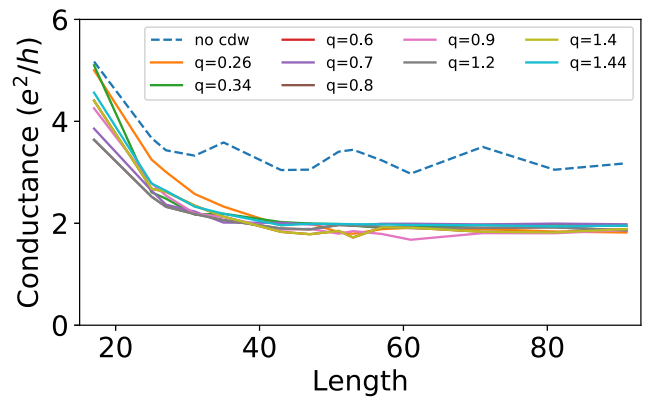

(b)

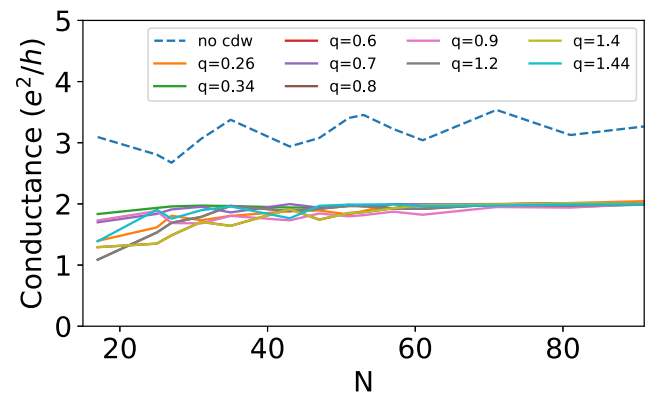

FIG. 11. Conductance at energy very close to $\omega / 2$ as a function of system size for different values of charge-density-wave perturbation for a sample of (a) width $W=51$ and (b) length $L=$ width $W=N$. This perturbation suppresses the contribution of bulk gapless modes, and thus only the quantized contribution from gapless edge modes survives.

(a)

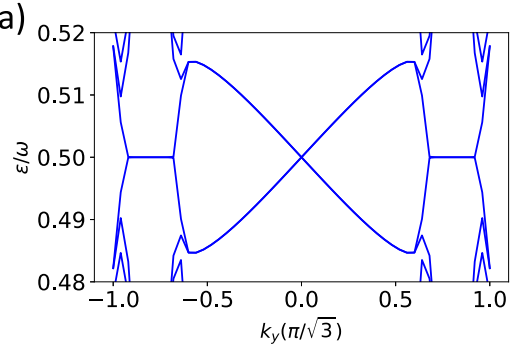

(b)

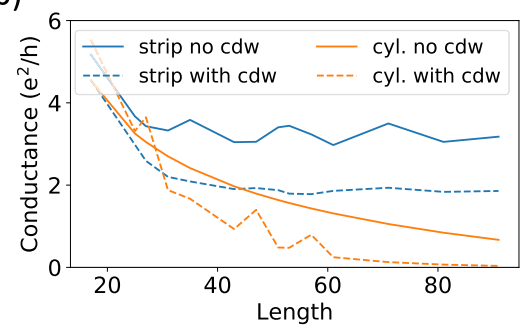

(c)

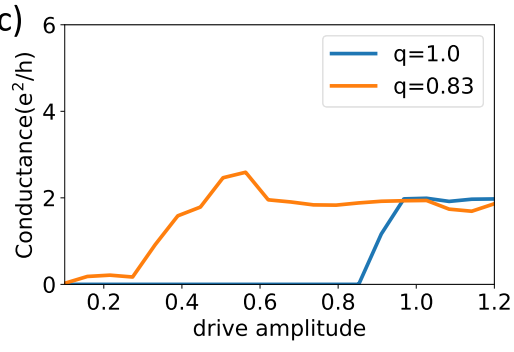

FIG. 12. (a) Spectra for a system with periodic boundary conditions in the $y$ direction. In this case, the translational vector is given by $\mathbf{a}_{2}=\sqrt{3} \hat{y}$. (b) Conductance as a function of sample length for two different geometries of width $W=51$ for a CDW of strength $A=0.5$ and wave vector $q=0.6$. (c) Conductance as a function of drive strength for two different CDW $q$ for a sample of length $L=81$ and width $W=51$. Cyl., cylinder. 

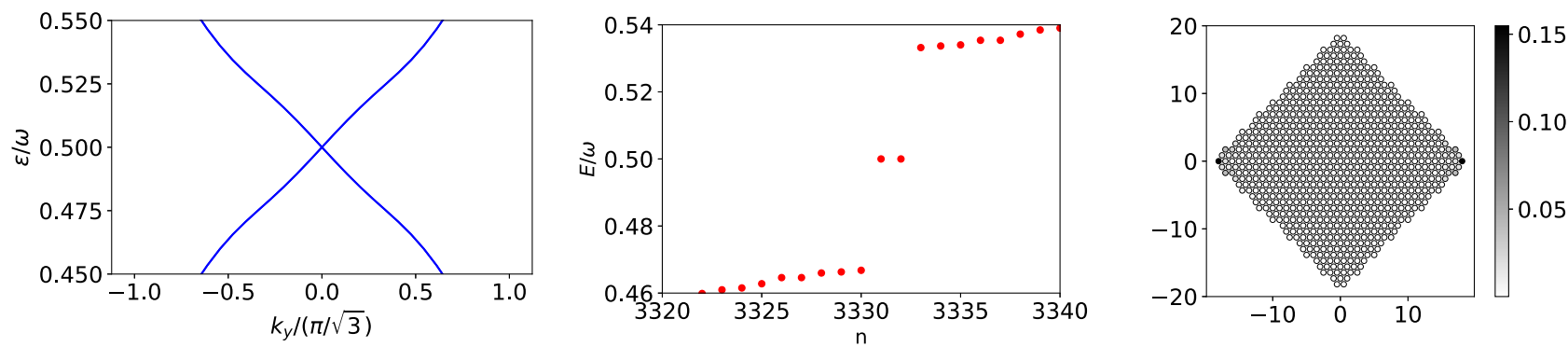

FIG. 13. Left: Band structure around $\omega / 2$ for the Floquet Hamiltonian (up to two Floquet sectors) with imaginary hopping term of Eq. (7) for periodic BCs in the $y$ direction. Center: Energy spectrum of the Floquet Hamiltonian with $H_{1}$ of Eq. (C4) around quasienergy $\omega / 2$ for open boundary conditions with reflection-symmetry-breaking term. Right: Support for the hinge mode for these boundary conditions corresponding to quasienergy $\omega / 2$. The parameters for this figure are $t_{a}=2.4, t_{b}=1.2, t_{3}=0.5, t_{w}=0.1, \beta_{0}=0.1, \gamma_{0}=0.8, \lambda_{0}=0.8$, and $t_{w_{2}}=0.1$.
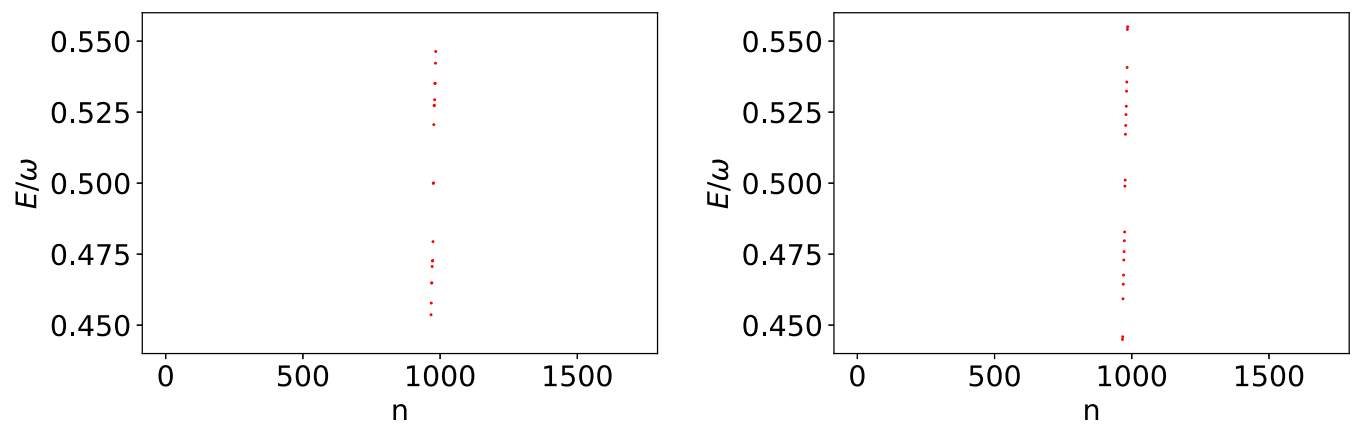

FIG. 14. Spectra around $\omega / 2$ for sample size $L=6$. Left: without disorder. Right: for a disorder with $d=1.0$.
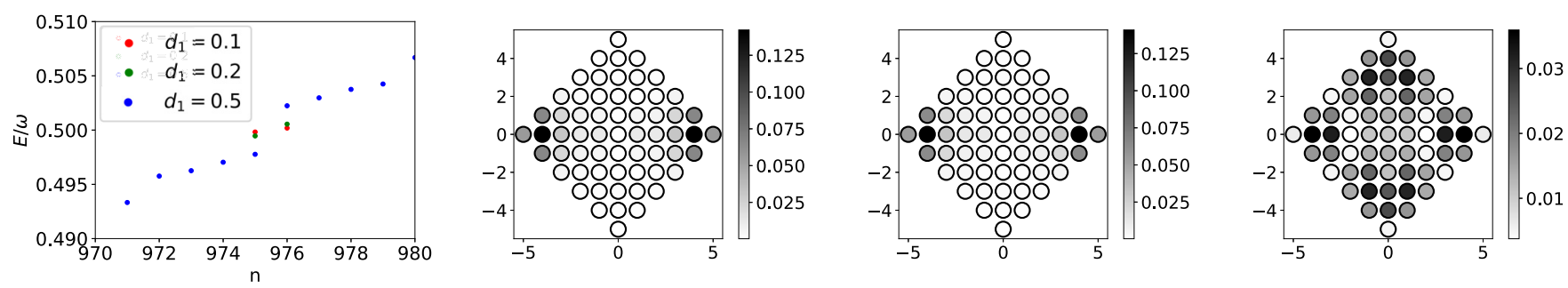

FIG. 15. Spectra around $\omega / 2$ for three different values of $d_{1}$ and corner modes associated with these states for sample size $L=6$. As $d_{1}$ increases, the corner modes start to mix with the bulk states. 

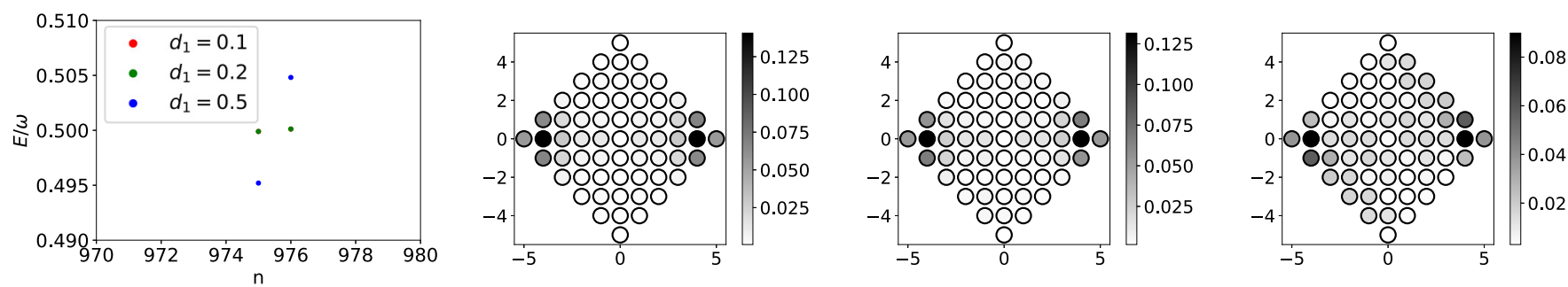

FIG. 16. Spectra around $\omega / 2$ for three different values of $d_{1}$ and corner modes associated with these states for sample size $L=6$. As $d_{1}$ increases, the corner modes start to mix with the bulk states. It is worth noticing that this splitting is much smaller than the separation between zero modes and the bulk modes, which is roughly equal to $0.02 \omega$.
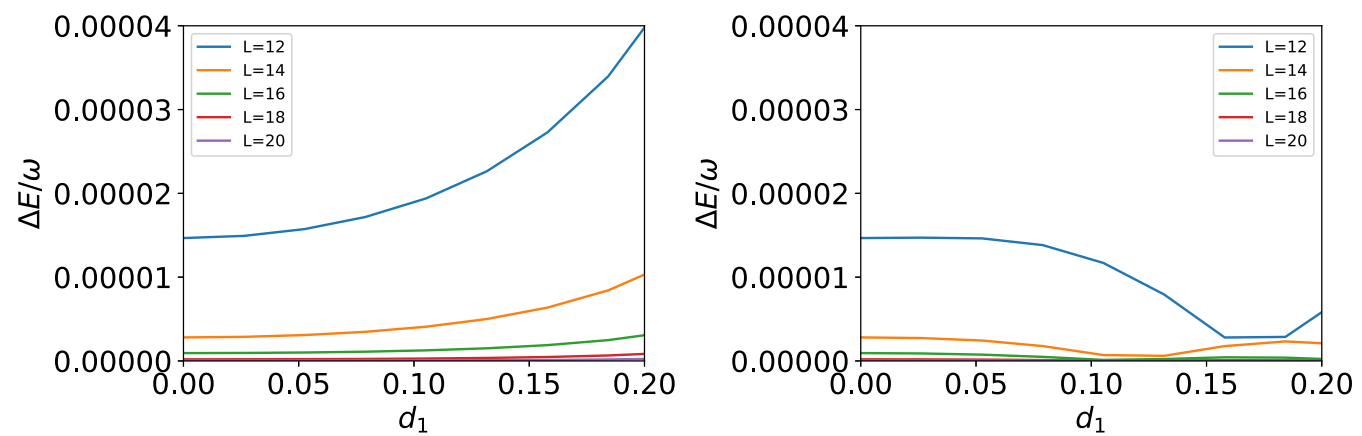

FIG. 17. Splitting of corner modes at quasienergy $\omega / 2$ as a function of perturbation strength $d_{1}$ for Hamiltonians in Eqs. (D1) and (D2), respectively, for different lengths of sample.
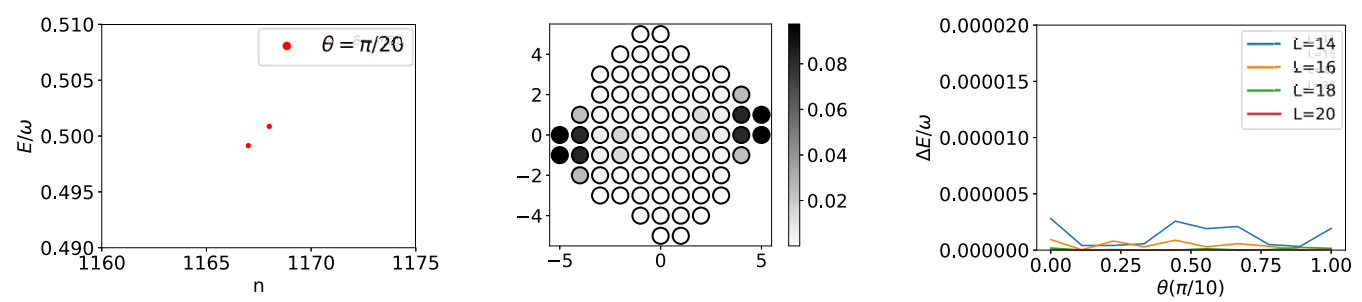

FIG. 18. Spectra around $\omega / 2$ for a sample rotated by angle $\theta$ about the $y$ axis. We notice that a change in orientation results in splitting of corner modes and the system can exhibit corner states for small values of $\theta$. The right panel shows how the splitting of Majorana modes is affected by the rotation angle $\theta$ for different lengths of sample. 
using KWANT [47]. Without this charge-density-wave term, the observed conductance has a contribution from both edge and bulk modes at quasienergy $\omega / 2$, but this term suppresses the bulk contribution as shown in Fig. 11. In the presence of the charge-density-wave perturbation, the conductance is quantized to 2, which indicates that it arises from the protected edge modes in the presence of a gapped bulk. This is further verified by the fact that modifying the geometry of the system from a strip to a cylindrical tube kills the contribution of edge modes and the conductance becomes zero as shown in Fig. 12. In order to calculate the conductance of the Floquet Hamiltonian, we first get the $S$ matrix using KWANT for two leads by treating the photon degree as a static degree of freedom, but since the lead is static, in order to calculate the transmission coefficient, we sum up over only those incoming modes that belong to the zeroth Floquet sector. For the outgoing modes, we keep nonzero photon sectors to account for photon-assisted transport [46], and thus the transmission at energy $E$ is given by

$$
T(E)=\sum_{\alpha, \beta, n}\left|S_{\alpha, n ; \beta, 0}(E)\right|^{2},
$$

where $\alpha$ and $\beta$ represent the outgoing and incoming modes, respectively, and $n$ is the photon degree of freedom.

\section{Phonon-induced complex hopping: Second-order topological insulator}

For class AIII, the static Hamiltonian

$$
\begin{aligned}
H_{0}(\mathbf{k})= & t_{a} \tau_{x}+2 t_{b}\left(\cos \frac{k_{x}}{2} \cos \frac{\sqrt{3} k_{y}}{2} \tau_{x}+\cos \frac{k_{x}}{2} \sin \frac{\sqrt{3} k_{y}}{2} \tau_{y}\right) \\
& +\left(t_{w} \cos \frac{k_{x}}{2} \cos \frac{\sqrt{3} k_{y}}{2}+\frac{t_{w_{2}}}{2} \cos \sqrt{3} k_{y}\right)\left(\sigma_{x} \tau_{x}-\sigma_{y} \tau_{y}\right) \\
& +\left(t_{w} \cos \frac{k_{x}}{2} \sin \frac{\sqrt{3} k_{y}}{2}+\frac{t_{w_{2}}}{2} \sin \sqrt{3} k_{y}\right)\left(\tau_{x} \sigma_{y}+\tau_{y} \sigma_{x}\right) \\
& +t_{3}\left(\tau_{x} \sigma_{x}+\sigma_{y} \tau_{y}\right)
\end{aligned}
$$

and phonon drive

$$
\begin{aligned}
H(\mathbf{k}, t)= & \beta(t)\left(-\sin \frac{k_{x}}{2} \cos \frac{\sqrt{3} k_{y}}{2} \tau_{y}+\sin \frac{k_{x}}{2} \sin \frac{\sqrt{3} k_{y}}{2} \tau_{x}\right) \\
& +\gamma(t) \sin \frac{k_{x}}{2} \cos \frac{\sqrt{3} k_{y}}{2}\left(\sigma_{x} \tau_{y}+\sigma_{y} \tau_{x}\right) \\
& +\gamma(t) \sin \frac{k_{x}}{2} \sin \frac{\sqrt{3} k_{y}}{2}\left(\sigma_{x} \tau_{x}-\sigma_{y} \tau_{y}\right),
\end{aligned}
$$

we obtained gapless edge modes which coexist with some gapless bulk modes. In the main text, we presented a CDW-based mechanism to remove these gapless points. Alternatively, as shown in Fig. 13, the gapless bulk modes can also be removed by a drive-induced interlayer imaginary hopping between dimers

$$
H_{I}(t)=\lambda(t) \sum\left(i a_{1, \mathbf{r}_{i}}^{\dagger} b_{2, \mathbf{r}_{i}+\mathbf{a}_{1}}+i a_{1, \mathbf{r}_{i}}^{\dagger} b_{2, \mathbf{r}_{i}+\mathbf{a}_{2}}+\text { H.c. }\right),
$$

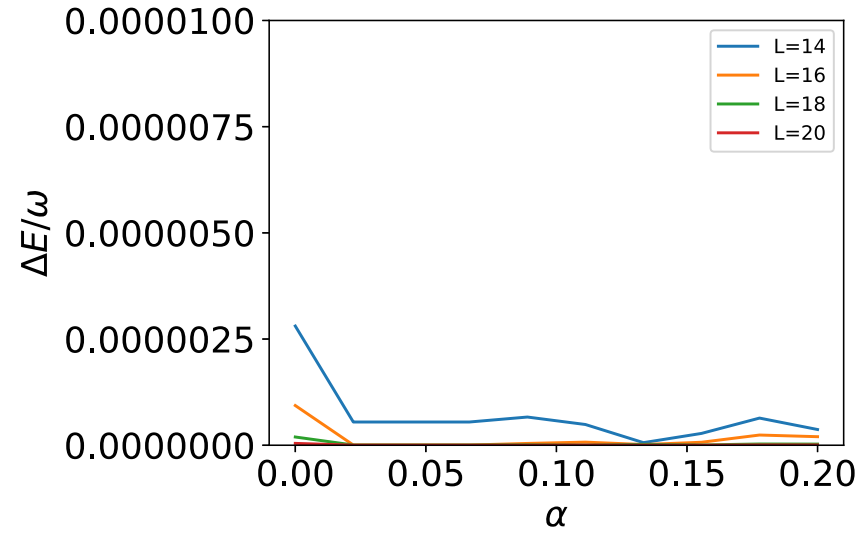

FIG. 19. Spectra around $\omega / 2$ for a sample where the upper two edges have slope equal to unity but the lower two edges have slope equal to $1-\alpha$. We notice that a change in slope for the lower edges results in splitting of corner modes and the system can exhibit corner states for small values of $\theta$. This figure shows how the splitting of Majorana modes is affected by the slope of the lower edges for different lengths of sample.

which in $k$ space becomes

$$
H_{I}(\mathbf{k})=\lambda(t) \cos \frac{k_{x}}{2} \sin \frac{\sqrt{3} k_{y}}{2}\left(\sigma_{x} \tau_{x}+\sigma_{y} \tau_{y}\right) .
$$

This kind of hopping might not be so easy to realize, but it verifies the fact that these gapless bulk modes are not protected by reflection symmetry alone. In this case, if we modify the boundary such that it gives rise to an effective reflectionsymmetry-breaking mass term, the gapless edge modes are replaced by the hinge modes on reflection-symmetric corners as shown in Fig. 13.

\section{APPENDIX D: EFFECT OF DISORDER AND OTHER PERTURBATIONS ON CORNER MODES OF CLASS D}

We study the effect of three different kinds of perturbations on Floquet Majorana corner modes discussed in the main text. We obtained these corner modes for open boundary conditions on a square-shaped sample of length $L$.

\section{Disorder}

We modify each nearest-neighbor interaction $t \rightarrow t(1+$ $h)$, where $h$ is a random number in the range $\left[-\frac{d}{2}, \frac{d}{2}\right]$. When $d \ll 1$, we observe that the corner modes persist although there degeneracy is slightly lifted as shown in Figs. 4 and 14. For larger disorder these states start to mix with bulk states, which results in energy splitting of zero modes. However, this splitting decreases significantly for a large-enough system size as shown in Fig. 5.

\section{Perturbations to the static Hamiltonian}

We first consider a perturbation which modified the static term:

$$
H_{0}(\mathbf{k}) \rightarrow H_{0}(\mathbf{k})+d_{1}\left(\sin k_{x} \sigma_{x} \tau_{z}+\sin k_{y} \sigma_{y} \tau_{z}\right) .
$$

In this case, the corner modes survive only for $d_{1}<0.5$ as shown in Fig. 15. 
Next, we consider a perturbation which modified the static term:

$$
H_{0}(\mathbf{k}) \rightarrow H_{0}(\mathbf{k})+d_{1}\left(\sin k_{x} \sigma_{y} \tau_{z}-\sin k_{y} \sigma_{x} \tau_{z}\right) .
$$

We observe that the corner modes can persist for small values of $d_{1}$ as shown in Fig. 16. As we increase $d_{1}$, we notice that the corner modes smear out and mix with bulk modes for large $d_{1}$. However, again this splitting is exponentially suppressed for large-enough system size as shown in Fig. 17.

\section{Modified corner}

We modify the corner by rotating the sample, and we notice that a small change in orientation can split up these modes as shown in Fig. 18.

We also tried to modify the corner conditions by changing the slope of the lower edges but keeping the rotation angle $\theta=0$. This kind of change results in a corner angle different from $\pi / 4$, and in Fig. 19 we show that the splitting of Majorana corner modes is not affected much by this change.
[1] A. P. Schnyder, S. Ryu, A. Furusaki, and A. W. W. Ludwig, Classification of topological insulators and superconductors in three spatial dimensions, Phys. Rev. B 78, 195125 (2008).

[2] A. Kitaev, Periodic table for topological insulators and superconductors, in Advances in Theoretical Physics: Landau Memorial Conference, AIP Conf. Proc. No. 1134 (AIP, Melville, NY, 2009), p. 22.

[3] S. Ryu, A. P. Schnyder, A. Furusaki, and A. W. W. Ludwig, Topological insulators and superconductors: Tenfold way and dimensional hierarchy, New J. Phys. 12, 065010 (2010).

[4] A. M. Turner, Y. Zhang, and A. Vishwanath, Entanglement and inversion symmetry in topological insulators, Phys. Rev. B 82, 241102(R) (2010).

[5] T. L. Hughes, E. Prodan, and B. A. Bernevig, Inversionsymmetric topological insulators, Phys. Rev. B 83, 245132 (2011).

[6] L. Fu, Topological Crystalline Insulators, Phys. Rev. Lett. 106, 106802 (2011).

[7] C.-K. Chiu, H. Yao, and S. Ryu, Classification of topological insulators and superconductors in the presence of reflection symmetry, Phys. Rev. B 88, 075142 (2013).

[8] K. Shiozaki and M. Sato, Topology of crystalline insulators and superconductors, Phys. Rev. B 90, 165114 (2014).

[9] J. C. Y. Teo and C. L. Kane, Topological defects and gapless modes in insulators and superconductors, Phys. Rev. B 82, 115120 (2010).

[10] C.-K. Chiu, J. C. Y. Teo, A. P. Schnyder, and S. Ryu, Classification of topological quantum matter with symmetries, Rev. Mod. Phys. 88, 035005 (2016).

[11] E. Khalaf, Higher-order topological insulators and superconductors protected by inversion symmetry, Phys. Rev. B 97, 205136 (2018).

[12] E. Khalaf, H. C. Po, A. Vishwanath, and H. Watanabe, Symmetry Indicators and Anomalous Surface States of Topological Crystalline Insulators, Phys. Rev. X 8, 031070 (2018).

[13] L. Trifunovic and P. W. Brouwer, Higher-Order Bulk-Boundary Correspondence for Topological Crystalline Phases, Phys. Rev. X 9, 011012 (2019).

[14] M. Z. Hasan and C. L. Kane, Colloquium: Topological insulators, Rev. Mod. Phys. 82, 3045 (2010).

[15] X.-L. Qi and S.-C. Zhang, Topological insulators and superconductors, Rev. Mod. Phys. 83, 1057 (2011).

[16] B. A. Bernevig and T. L. Hughes, Topological Insulators and Topological Superconductors (Princeton University Press, Princeton, NJ, 2013).
[17] W. A. Benalcazar, B. A. Bernevig, and T. L. Hughes, Electric multipole moments, topological multipole moment pumping, and chiral hinge states in crystalline insulators, Phys. Rev. B 96, 245115 (2017).

[18] Y. Peng, Y. Bao, and F. von Oppen, Boundary Green functions of topological insulators and superconductors, Phys. Rev. B 95 , 235143 (2017).

[19] J. Langbehn, Y. Peng, L. Trifunovic, F. von Oppen, and P. W. Brouwer, Reflection-Symmetric Second-Order Topological Insulators and Superconductors, Phys. Rev. Lett. 119, 246401 (2017).

[20] W. A. Benalcazar, B. A. Bernevig, and T. L. Hughes, Quantized electric multipole insulators, Science 357, 61 (2017).

[21] Z. Song, Z. Fang, and C. Fang, $(d-2)$-Dimensional Edge States of Rotation Symmetry Protected Topological States, Phys. Rev. Lett. 119, 246402 (2017).

[22] F. Schindler, A. M. Cook, M. G. Vergniory, Z. Wang, S. S. P. Parkin, B. A. Bernevig, and T. Neupert, Higher-order topological insulators, Sci. Adv. 4, eaat0346 (2018).

[23] M. Geier, L. Trifunovic, M. Hoskam, and P. W. Brouwer, Second-order topological insulators and superconductors with an order-two crystalline symmetry, Phys. Rev. B 97, 205135 (2018).

[24] Z. Yan, F. Song, and Z. Wang, Majorana Corner Modes in a High-Temperature Platform, Phys. Rev. Lett. 121, 096803 (2018).

[25] R. W. Bomantara and J. Gong, Measurement-only quantum computation with Floquet Majorana corner modes, Phys. Rev. B 101, 085401 (2020).

[26] S. Imhof, C. Berger, F. Bayer, J. Brehm, L. W. Molenkamp, T. Kiessling, F. Schindler, C. H. Lee, M. Greiter, T. Neupert, and R.Thomale, Topolectrical-circuit realization of topological corner modes, Nat. Phys. 14, 925 (2018).

[27] C. W. Peterson, W. A. Benalcazar, T. L. Hughes, and G. Bahl, A quantized microwave quadrupole insulator with topologically protected corner states, Nature (London) 555, 346 (2018).

[28] M. Serra-Garcia, V. Peri, R. Süsstrunk, O. R. Bilal, T. Larsen, L. G. Villanueva, and S. D. Huber, Observation of a phononic quadrupole topological insulator, Nature (London) 555, 342 (2018).

[29] H. Xue, Y. Yang, F. Gao, Y. Chong, and B. Zhang, Acoustic higher-order topological insulator on a Kagome lattice, Nat. Mater. 18, 108 (2019).

[30] X. Ni, M. Weiner, A. Alù, and A. B. Khanikaev, Observation of higher-order topological acoustic states protected by generalized chiral symmetry, Nat. Mater. 18, 113 (2019). 
[31] F. Schindler, Z. Wang, M. G. Vergniory, A. M. Cook, A. Murani, S. Sengupta, A. Y. Kasumov, R. Deblock, S. Jeon, I. Drozdov, H. Bouchiat, S. Guéron, A. Yazdani, B. A. Bernevig, and T. Neupert, Higher-order topology in bismuth, Nat. Phys. 14, 918 (2018).

[32] T. Morimoto, H. C. Po, and A. Vishwanath, Floquet topological phases protected by time glide symmetry, Phys. Rev. B 95, 195155 (2017).

[33] Y. Peng and G. Refael, Floquet Second-Order Topological Insulators from Nonsymmorphic Space-Time Symmetries, Phys. Rev. Lett. 123, 016806 (2019).

[34] Y. Peng, Floquet higher-order topological insulators and superconductors with space-time symmetries, Phys. Rev. Res. 2, 013124 (2020).

[35] A. V. Kuznetsov and C. J. Stanton, Theory of Coherent Phonon Oscillations in Semiconductors, Phys. Rev. Lett. 73, 3243 (1994).

[36] H. Hübener, U. De Giovannini, and A. Rubio, Phonon driven Floquet matter, Nano Lett. 18, 1535 (2018).

[37] H. L. Calvo, J. S. Luna, V. Dal Lago, and L. E. F. Foa Torres, Robust edge states induced by electron-phonon interaction in graphene nanoribbons, Phys. Rev. B 98, 035423 (2018).

[38] R. W. Bomantara, L. Zhou, J. Pan, and J. Gong, Coupled-wire construction of static and Floquet second-order topological insulators, Phys. Rev. B 99, 045441 (2019).

[39] M. Rodriguez-Vega, A. Kumar, and B. Seradjeh, Higher-order Floquet topological phases with corner and bulk bound states, Phys. Rev. B 100, 085138 (2019).

[40] S. Konschuh, M. Gmitra, and J. Fabian, Tight-binding theory of the spin-orbit coupling in graphene, Phys. Rev. B 82, 245412 (2010).

[41] H. Min, J. E. Hill, N. A. Sinitsyn, B. R. Sahu, L. Kleinman, and A. H. MacDonald, Intrinsic and Rashba spin-orbit interactions in graphene sheets, Phys. Rev. B 74, 165310 (2006).

[42] J. C. Slater and G. F. Koster, Simplified LCAO method for the periodic potential problem, Phys. Rev. 94, 1498 (1954).

[43] A. Subedi, A. Cavalleri, and A. Georges, Theory of nonlinear phononics for coherent light control of solids, Phys. Rev. B 89, 220301(R) (2014).

[44] M. Gu and J. M. Rondinelli, Nonlinear phononic control and emergent magnetism in Mott insulating titanates, Phys. Rev. B 98, 024102 (2018).

[45] J.-M. Jancu, R. Scholz, F. Beltram, and F. Bassani, Empirical spds* tight-binding calculation for cubic semiconductors: General method and material parameters, Phys. Rev. B 57, 6493 (1998).

[46] M. V. Moskalets, Scattering Matrix Approach to Non-stationary Quantum Transport (World Scientific, Singapore, 2012).

[47] C. W. Groth, M. Wimmer, A. R. Akhmerov, and X. Waintal, Kwant: A software package for quantum transport, New J. Phys. 16, 063065 (2014).

[48] R. I. Tobey, D. Prabhakaran, A. T. Boothroyd, and A. Cavalleri, Ultrafast Electronic Phase Transition in $\mathrm{La}_{1 / 2} \mathrm{Sr}_{3 / 2} \mathrm{MnO}_{4}$ by
Coherent Vibrational Excitation: Evidence for Nonthermal Melting of Orbital Order, Phys. Rev. Lett. 101, 197404 (2008).

[49] T. L. Cocker, V. Jelic, M. Gupta, S. J. Molesky, J. A. J. Burgess, G. De Los Reyes, L. V. Titova, Y. Y. Tsui, M. R. Freeman, and F. A. Hegmann, An ultrafast terahertz scanning tunneling microscope, Nat. Photonics 7, 620 (2013).

[50] J. W. McIver, B. Schulte, F.-U. Stein, T. Matsuyama, G. Jotzu, G. Meier, and A. Cavalleri, Light-induced anomalous Hall effect in graphene, Nat. Phys. 16, 38 (2020).

[51] S. Mathias, M. Wiesenmayer, F. Deicke, A. Ruffing, L. MiajaAvila, M. M. Murnane, H. C. Kapteyn, M. Bauer, and M. Aeschlimann, Time and angle resolved photoemission spectroscopy using femtosecond visible and high-harmonic light, in The LXIII Yamada Conference on Photo-Induced Phase Transition and Cooperative Phenomena, 11-15 November 2008, Osaka, Japan, Journal of Physics Conference Series Vol. 148 (IOP, London, 2009), p. 012042.

[52] F. Schmitt, P. S. Kirchmann, U. Bovensiepen, R. G. Moore, J. H. Chu, D. H. Lu, L. Rettig, M. Wolf, I. R. Fisher, and Z. X. Shen, Ultrafast electron dynamics in the charge density wave material $\mathrm{TbTe}_{3}$, New J. Phys. 13, 063022 (2011).

[53] J. A. Sobota, S. Yang, J. G. Analytis, Y. L. Chen, I. R Fisher, P. S. Kirchmann, and Z.-X. Shen, Ultrafast Optical Excitation of a Persistent Surface-State Population in the Topological Insulator $\mathrm{Bi}_{2} \mathrm{Se}_{3}$, Phys. Rev. Lett. 108, 117403 (2012).

[54] H. Soifer, A. Gauthier, A. F. Kemper, C. R. Rotundu, S.-L. Yang, H. Xiong, D. Lu, M. Hashimoto, P. S. Kirchmann, J. A. Sobota, and Z.-X. Shen, Band-Resolved Imaging of Photocurrent in a Topological Insulator, Phys. Rev. Lett. 122, 167401 (2019).

[55] E. J. Sie, T. Rohwer, C. Lee, and N. Gedik, Time-resolved XUV ARPES with tunable 24-33 eV laser pulses at $30 \mathrm{meV}$ resolution, Nat. Commun. 10, 3535 (2019).

[56] A. P. Cote, A. I. Benin, N. W. Ockwig, M. O'Keeffe, A. J. Matzger, and O. M. Yaghi, Porous, crystalline, covalent organic frameworks, Science 310, 1166 (2005).

[57] W. Jiang, H. Huang, and F. Liu, A Lieb-like lattice in a covalent-organic framework and its Stoner ferromagnetism, Nat. Commun. 10, 2207 (2019).

[58] T. W. Kim, S. Jun, Y. Ha, R. K. Yadav, A. Kumar, C.-Y. Yoo, I. Oh, H.-K. Lim, J. W. Shin, R. Ryoo, H. Kim, J. Kim, J.-O. Baeg, and $\mathrm{H}$. Ihee, Ultrafast charge transfer coupled with lattice phonons in two-dimensional covalent organic frameworks, Nat. Commun. 10, 1873 (2019).

[59] J. M. B. Lopes dos Santos, N. M. R. Peres, and A. H. Castro Neto, Graphene Bilayer with a Twist: Electronic Structure, Phys. Rev. Lett. 99, 256802 (2007).

[60] A. I. Cocemasov, D. L. Nika, and A. A. Balandin, Phonons in twisted bilayer graphene, Phys. Rev. B 88, 035428 (2013).

Correction: The affiliation indicators for the third author were ordered incorrectly and have been fixed. 